This item was submitted to Loughborough's Research Repository by the author.

Items in Figshare are protected by copyright, with all rights reserved, unless otherwise indicated.

\title{
Subterahertz Chaos Generation by Coupling a Superlattice to a Linear Resonator
}

\section{PLEASE CITE THE PUBLISHED VERSION}

http://dx.doi.org/10.1103/PhysRevLett.112.116603

\section{PUBLISHER}

(C) American Physical Society

\section{VERSION}

VoR (Version of Record)

\section{PUBLISHER STATEMENT}

This work is made available according to the conditions of the Creative Commons Attribution-NonCommercialNoDerivatives 4.0 International (CC BY-NC-ND 4.0) licence. Full details of this licence are available at: https://creativecommons.org/licenses/by-nc-nd/4.0/

\section{LICENCE}

CC BY-NC-ND 4.0

\section{REPOSITORY RECORD}

Hramov, Alexander E., V.V. Makarov, Alexey A. Koronovskii, S.A. Kurkin, Marat Gaifullin, Natalia V. Alexeeva, Kirill Alekseev, et al.. 2014. "Subterahertz Chaos Generation by Coupling a Superlattice to a Linear Resonator”. Loughborough University. https://hdl.handle.net/2134/17299. 


\title{
Subterahertz Chaos Generation by Coupling a Superlattice to a Linear Resonator
}

\author{
A. E. Hramov, ${ }^{1,4}$ V. V. Makarov, ${ }^{1}$ A. A. Koronovskii, ${ }^{1,4}$ S. A. Kurkin, ${ }^{1}$ M. B. Gaifullin, ${ }^{2}$ N. V. Alexeeva, ${ }^{2}$ K. N. Alekseev, ${ }^{2}$ \\ M. T. Greenaway, ${ }^{3}$ T. M. Fromhold, ${ }^{3}$ A. Patanè, ${ }^{3}$ F. V. Kusmartsev, ${ }^{2, *}$ V. A. Maksimenko, ${ }^{4}$ \\ O. I. Moskalenko, ${ }^{4}$ and A. G. Balanov ${ }^{2}$ \\ ${ }^{1}$ Faculty of Nonlinear Processes, Saratov State University, Astrakhanskaya 83, Saratov 410012, Russia \\ ${ }^{2}$ Department of Physics, Loughborough University, Loughborough LE11 3TU, United Kingdom \\ ${ }^{3}$ School of Physics and Astronomy, University of Nottingham, Nottingham NG7 2RD, United Kingdom \\ ${ }^{4}$ Saratov State Technical University, Politechnicheskaja 77, Saratov 410054, Russia
}

(Received 12 July 2013; published 18 March 2014)

\begin{abstract}
We investigate the effects of a linear resonator on the high-frequency dynamics of electrons in devices exhibiting negative differential conductance. We show that the resonator strongly affects both the dc and ac transport characteristics of the device, inducing quasiperiodic and high-frequency chaotic current oscillations. The theoretical findings are confirmed by experimental measurements of a GaAs/AlAs miniband semiconductor superlattice coupled to a linear microstrip resonator. Our results are applicable to other active solid state devices and provide a generic approach for developing modern chaos-based high-frequency technologies including broadband chaotic wireless communication and superfast random-number generation.
\end{abstract}

The interaction of matter with electromagnetic (EM) waves confined within a resonator remains one of the most important and widespread problems in physics. This generic system has many implications in different areas of science including cold atoms [1], quantum engineering [2], metamaterials [3], and $\mathrm{THz}$ and nanoscale lasers with submillimeter wavelength cavities [4,5]. In electronics and optics, resonators are often used to enhance the generated power [4] or to tune the frequency of radiated EM waves [6]. The action of a high quality resonator may also provide a way to achieve monochromatization and coherence purification of electromagnetic output [7].

Devices that exhibit negative differential conductance (NDC) have great potential to operate in the technologically important $\mathrm{GHz}-\mathrm{THz}$ frequency range, even at room temperature. At high carrier density [8], the NDC triggers the formation of propagating charge domains [9], and active EM properties. This includes an ability to amplify an injected high frequency signal [10]. Here we focus on a semiconductor superlattice (SL), where the physical mechanism for NDC is the onset of Bloch oscillations in a single miniband [11-14]. This type of device is different from quantum cascade lasers (QCLs) [15], used as sources of THz radiation [16-20].

The dynamics of charge domains in miniband SLs can be effectively controlled by applying alternating electric or tilted magnetic fields [21-23]. The propagating and periodically pulsing charge domains in GaAs/AlGaAs SLs produce a powerful high-frequency $\mathrm{GHz}$ radiation [24] and very effective frequency multiplication up to several $\mathrm{THz}$ [25]. However, the influence of EM resonators on the electron dynamics in SLs is still largely unexplored [26].

To address this question we theoretically and experimentally study how a linear resonator induces highfrequency chaotic current oscillations in a SL. Such oscillations with a frequency up to $400 \mathrm{GHz}$ appear in multilayer semiconductor structures made of reverse $p$ - $n$ junctions [27]. They arise due to nonlinearity associated with asymmetric doping. The nonlinearity can be also imposed by contacts, load lines, and even parasitic capacitances [28]. In our case, the uniformly doped miniband SL with Ohmic contacts generates only regular dynamics. However, we show, counterintuitively, that even a linear resonator can drive the regular oscillations chaotic. By changing the voltage applied to the coupled SL and resonator, one can switch between periodic, quasiperiodic, and chaotic current oscillations in SLs that exhibit only periodic oscillations in the absence of a resonator. Our theoretical analysis and experiments are in good quantitative agreement. The phenomena that we identify suggest applications for new resonant control of dynamics in solid state systems with NDC.

High-frequency chaotic generators are currently in strong demand in a number of modern key technologies including fast random-number generation (see [29] and references therein) and chaos-based communication systems [30-32]. However, in contrast to the optical frequency range, chaotic sub- and terahertz generators are still underdeveloped [29]. Our findings propose a generic approach to develop such generators.

We consider a SL interacting with a resonator, as shown schematically in Fig. 1(a). We assume that only one EM field mode is excited in the resonator, which has the eigenfrequency $f_{Q}$ and quality factor $Q$, and can be represented by the equivalent $R L C$ circuit shown in Fig. 1(b). The SL serves as a generator of electric current $I$, controlled by a voltage $V_{\mathrm{SL}}(t)$, dropped across the SL, which includes both the dc supply voltage $V_{0}$ and the ac voltage $V_{1}(t)$ generated by the RLC circuit.

To make our model realistic we follow the approach described in [23], with SL parameters taken from recent experiments $[21,22]$. The miniband transport region is 


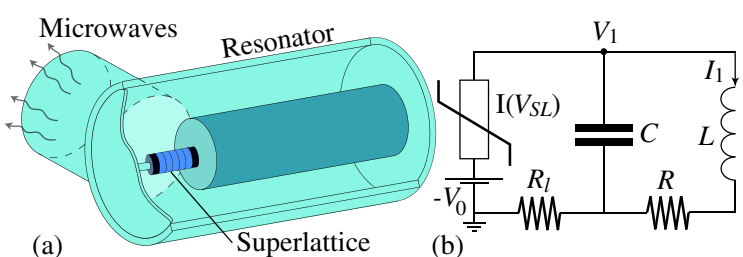

FIG. 1 (color online). (a) Schematic diagram of a semiconductor superlattice coupled to an external EM resonator. (b) Equivalent circuit for a SL interacting with an external single-mode resonator. Here $C, L$, and $R$ are the equivalent capacitance, inductance, and resistance of the resonator, $I\left(V_{\mathrm{SL}}\right)$ is the current through the SL, with voltage $V_{\mathrm{SL}}$ dropped across it, and $V_{0}$ is the dc supply voltage. The load resistance is $R_{l}=0.1 \Omega$.

discretized into $N=480$ layers, each of width $\delta x=0.24 \mathrm{~nm}$, small enough to approximate a continuum and ensure convergence of the numerical scheme. The discretized current continuity equation [33] is

$$
e \delta x \frac{d n_{m}}{d t}=J_{m-1}-J_{m}, \quad m=1, \ldots, N,
$$

where $e>0$ is the electron charge, $n_{m}$ is the charge density at the right-hand edge of the $m$ th layer, at position $x=m \delta x$, and $J_{m-1}$ and $J_{m}$ are the areal current densities at the leftand right-hand boundaries of the $m$ th layer

$$
J_{m}=e n_{m} v_{d}\left(\bar{F}_{m}\right),
$$

where $\bar{F}_{m}$ is the mean field in the $m$ th layer [23]. The drift velocity, $v_{d}(\bar{F})$, corresponding to the electric field, $\bar{F}$, can be calculated as in [34]:

$$
v_{d}=\frac{\Delta d}{2 \hbar} \frac{I_{1}\left(\Delta / 2 k_{B} T\right)}{I_{0}\left(\Delta / 2 k_{B} T\right)} \frac{e \bar{F} d \tau / \hbar}{1+(e \bar{F} d \tau / \hbar)^{2}},
$$

where $d=8.3 \mathrm{~nm}$ is the period of the $\mathrm{SL}, \Delta=19.1 \mathrm{meV}$ is the miniband width, $T=4.2 \mathrm{~K}$ is the temperature, $k_{B}$ is the Boltzmann constant and $I_{n}(x)$, where $n=0,1$, is a modified Bessel function of the first kind.

The electric fields $F_{m}$ and $F_{m+1}$ at the left- and righthand edges of the $m$ th layer, respectively, are related by the discretized Poisson equation [33]

$$
F_{m+1}=\frac{e \delta x}{\varepsilon_{0} \varepsilon_{r}}\left(n_{m}-n_{D}\right)+F_{m}, \quad m=1, \ldots, N,
$$

where $\varepsilon_{0}$ and $\varepsilon_{r}=12.5$ are, respectively, the absolute and relative permittivities and $n_{D}=3 \times 10^{22} \mathrm{~m}^{-3}$ is the $n$-type doping density in the SL layers [21]. The current density injected into the contact layers of the SL subjected to the field $F_{0}$ is $J_{0}=\sigma F_{0}$, where $\sigma=3788 \mathrm{~S} \mathrm{~m}^{-1}$ is the conductivity of the heavily doped emitter [23]. The voltage $V_{\mathrm{SL}}$ dropped across the SL defines a global constraint:

$$
V_{\mathrm{SL}}=U+\frac{\delta x}{2} \sum_{m=1}^{N}\left(F_{m}+F_{m+1}\right),
$$

where the voltage $U$ dropped across the contacts includes the effect of charge accumulation and depletion in the emitter and collector regions, and the voltage across the contact resistance [23], $R=17 \Omega$. Finally, the current through the device is

$$
I(t)=\frac{A}{N+1} \sum_{m=0}^{N} J_{m},
$$

where $A=5 \times 10^{-10} \mathrm{~m}^{2}$ is the cross-sectional area of the SL [13,21,23].

We apply Kirchoff's equations to the equivalent circuit shown in Fig. 1(b) and obtain the following equations:

$$
\begin{aligned}
& \frac{d V_{1}}{d t}=\frac{I\left(V_{\mathrm{SL}}\right)-I_{1}}{C}, \\
& \frac{d I_{1}}{d t}=\frac{V_{0}-V_{\mathrm{SL}}+R I_{1}+R_{l} I\left(V_{\mathrm{SL}}\right)}{L},
\end{aligned}
$$

where $V_{1}(t)$ and $I_{1}(t)$ are, respectively, the voltage across the capacitor and the current through the inductor [see Fig. 1(b)]. Thus, the voltage dropped across the SL is $V_{\mathrm{SL}}=V_{1}+V_{0}$. The eigenfrequency of the resonator is $f_{Q}=$ $1 /(2 \pi \sqrt{L C})$ and the quality factor is $Q=(1 / R) \sqrt{L / C}$.

The current through the SL is either constant or oscillates depending on the voltage $V_{0}$ applied to the circuit. If the $\mathrm{SL}$ is decoupled from the resonator $\left(V_{1}=0\right)$, its currentvoltage characteristic, $I\left(V_{\mathrm{SL}}\right)$, is of the Esaki-Tsu type [11] and the current oscillations are always periodic [23]. However, if the resonator is coupled to the SL, the circuit behavior changes greatly.

In the coupled regime, the $I\left(V_{\mathrm{SL}}\right)$ characteristic obtained by averaging the current $I(t)$ [Eq. (6)] over time is shown in Fig. 2 by the dark gray (red) curve, which has a form similar to one discussed in [35]. In both cases there are charge domains, whose instabilities or transformation lead to the appearance of specific features in the $I(V)$ curves. However, we consider miniband transport, while in Ref. [35] sequential tunneling is discussed.

For low voltages, the $I\left(V_{0}\right)$ curve is Ohmic and the current attains a maximum value when $V_{0}=V_{\text {crit }}$ (marked by the arrow in Fig. 2). Thereafter, the current tends to decrease with increasing $V_{0}$ due to the onset of Bloch oscillations. However, for $V_{0}>V_{\text {crit }}$ there is a series of peaks in $I\left(V_{0}\right)$, which do not occur in the absence of the resonator. These peaks correspond to the transitions between the periodic and chaotic dynamical regimes. To illustrate this, in Fig. 2 we superimpose a bifurcation diagram in which each point shows the local maximum value of $V_{1}(t)$ (RH scale) obtained for each $V_{0}$ value (omitting any initial transient behavior). Thus, for a given $V_{0}$, a single point in the bifurcation diagram represents either a steady state or periodic $V_{1}(t)$ oscillations. Similarly, several separated points indicate period-added oscillations and a complex set of points implies either quasiperiodic or chaotic behavior. Figure 2 reveals that each peak in the $I\left(V_{0}\right)$ curve corresponds to a transition between different types of dynamics in the bifurcation diagram. With 


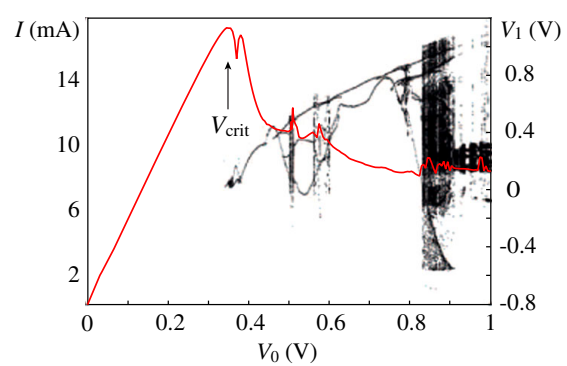

FIG. 2 (color online). $\quad I\left(V_{0}\right)$ (red curve, scale on the left-hand axis) calculated for a SL coupled to a resonator with $f_{Q}=$ $13.81 \mathrm{GHz}$ and $Q=150$. Overlaid is the bifurcation diagram (black dots) of the voltage oscillations $V_{1}(t)$ (right-hand axis) versus $V_{0}$. The critical value $V_{0}=V_{\text {crit }}$ for the onset of the calculated microwave generation is shown by the arrow.

increasing $V_{0}$, the dynamics changes first from a steady state solution to periodic oscillations when $V_{0} \approx V_{\text {crit }}$. Thereafter, increasing $V_{0}$ induces multiple transitions between periodic and aperiodic oscillations, with each transition corresponding to a peak in the $I\left(V_{0}\right)$ curve. For example, when $V_{0} \approx$ $0.5 \mathrm{~V}$ and $V_{0} \approx 0.83 \mathrm{~V}$, we find peaks in the $I\left(V_{0}\right)$ curve and a transition between periodic and chaotic dynamics. The presence of chaos is confirmed by calculation of the Lyapunov exponents [36] (see Fig. S1 in the Supplemental Material [37]). Note, the SL demonstrates a transition to chaos through intermittency with increase of the dc voltage $V_{0}$ (see Supplemental Material [37], Fig. S2).

Transitions between different oscillatory regimes can also be realized by fixing the parameters of the SL and varying those of the resonator. For example, Fig. 3(a) shows the bifurcation diagram obtained by varying the resonator eigenfrequency $f_{Q}$ when $V_{0}=0.54 \mathrm{~V}$. The variation of $f_{Q}$ produces a number of bifurcations leading to the appearance and disappearance of aperiodic oscillations. The regions of chaotic oscillations [shaded gray in Fig. 3(a)] are separated by a narrow region of periodic oscillations.

To gain further insight into transitions between the dynamical regimes, in Figs. 3(b), (c) we show the Fourier power spectra of the $V_{1}(t)$ oscillations for $f_{Q}$ values $f_{Q p}=$ 13.07 (b) and $f_{Q_{c}}=13.81 \mathrm{GHz}$ (c) [marked by vertical red dashed lines in Fig. 3(a)] before and after the onset of chaos at $f_{Q}=13.6 \mathrm{GHz}$ [upper arrow in Fig. 3(a)]. Figures 3(d) and 3(e) show the corresponding phase portraits of the oscillations $\left[V_{1}(t), V_{1}(t-\tau)\right]$ constructed by the Takens method (the method of delayed coordinates) [38] with time delay $\tau=0.3$ ps. Before the transition, when $f_{Q p}=13.07 \mathrm{GHz}$, the oscillations have a discrete spectrum [Fig. 3(b)] with equidistant peaks reflecting the periodic character of $V_{1}(t)$, which is confirmed by the closed phase trajectory [Fig. 3(d)]. After the transition, when $f_{Q c}=13.81 \mathrm{GHz}$, the frequency spectrum becomes continuous [Fig. 3(c)], and the trajectory in Fig. 3(e) fills the phase space, indicating chaotic dynamics. We emphasise that the appearance of chaos in a SL coupled to a linear resonator is surprising because chaos is a fundamentally nonlinear phenomenon. Note, the SL does not reveal chaos when it is decoupled from the resonator.
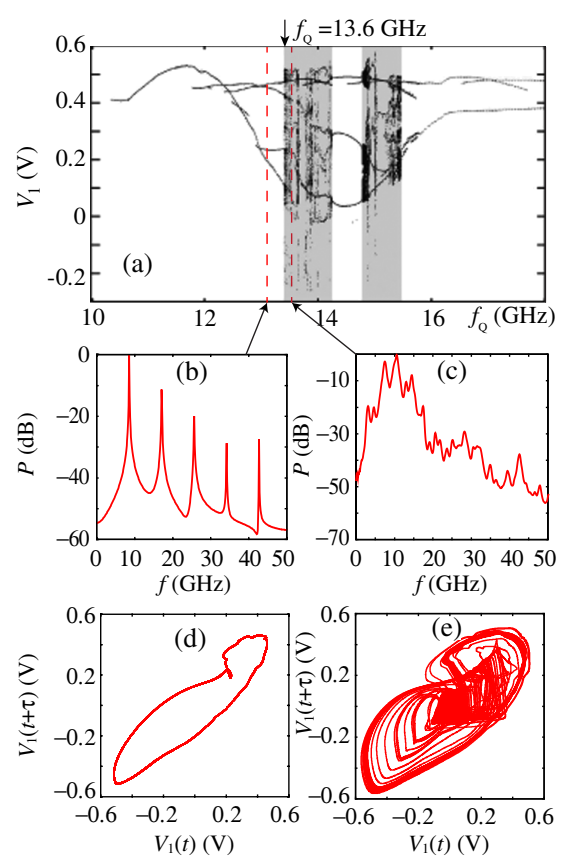

FIG. 3 (color online). (a) Bifurcation diagram showing local maxima $V_{1}$ of the voltage oscillations $V_{1}(t)$ versus $f_{Q}$ at $V_{0}=0.54 \mathrm{~V}$. The gray panels mark the $f_{Q}$ ranges where chaotic oscillations occur. (b),(c) Power spectra of voltage oscillations $V_{1}(t)$ for the resonator frequencies [vertical red dashed lines in (a)] $f_{Q p}=13.07$ (b) and $f_{Q c}=13.81 \mathrm{GHz}$ (c). The corresponding time-delayed phase trajectories are shown in (d) and (e).

In Fig. 4 we show the parameter space $\left(f_{Q}, Q\right)$, illustrating the regions of chaotic dynamics (shaded gray). The figure reveals that chaos is a robust phenomenon, which appears for a wide range of $f_{Q}$ values and depends critically on the $Q$ factor of the resonator. Chaotic oscillations only appear when $Q$ exceeds a threshold value, which, for $V_{0}=0.54 \mathrm{~V}$, is $\approx 50$. Increasing $Q$ above 50 , the width of the chaotic region in $f_{Q}$ initially increases and then saturates for $Q>150$. Since the frequency of the current oscillations in the decoupled SL is $f_{\mathrm{SL}}=$ $16.76 \mathrm{GHz}$ when $V_{0}=0.54 \mathrm{~V}$, we conclude that chaos occurs when $f_{Q}$ is well detuned from $f_{\mathrm{SL}}$.

It is also important to consider how any parasitic capacitance and inductance associated with the wires and contacts to SL devices affects the system dynamics. To study this we consider an equivalent circuit, which takes into account this parasitic reactance in addition to the influence of an external resonator (see [37], Fig. S3 for an equivalent circuit of a SL coupled to two resonators). Our calculations reveal that this system can also exhibit chaotic oscillations. This is shown in Figs. 5(a)-(c) where a transition from periodic (a) to quasiperiodic (b), (c) current oscillations occurs with increasing $V_{0}$. Here, the parasitic resonator is characterized by the quality factor $Q_{1}=20$ and eigenfrequency $f_{Q 1}=2.82 \mathrm{GHz}$ and the external resonator has parameters $Q_{2}=37.6$ and $f_{Q 2}=3.1 \mathrm{GHz}$.

When $V_{0}=0.42 \mathrm{~V}$ [Fig. 5(a)] the system exhibits periodic $V_{1}(t)$ oscillations (right panel) with a single dominant 


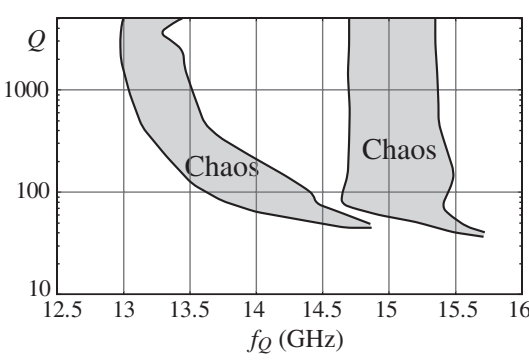

FIG. 4. Parameter plane $\left(f_{Q}, Q\right)$ showing where the $I(t)$ and $V_{1}(t)$ oscillations are chaotic (gray) or periodic (white) when $V_{0}=0.54 \mathrm{~V}$.

frequency $\approx 3.5 \mathrm{GHz}$ in the Fourier power spectrum (left panel). Increasing $V_{0}$ to $0.43 \mathrm{~V}$ [Fig. 5(b)] changes the character of the oscillations: the Fourier spectrum starts to show peaks at combination frequencies (left panel), and the $V_{1}(t)$ oscillations become quasiperiodic (right panel). Finally, by increasing $V_{0}$ to $0.44 \mathrm{~V}$ [Fig. 5(c)] we find that the $V_{1}(t)$ oscillations (right panel) are chaotic with a broad spectrum (left panel). This evolution of the $V_{1}(t)$ curves and Fourier spectra suggests that chaos appears via the breakdown of quasiperiodicity [39].

To verify these predictions, we performed experimental measurements and complementary numerical modelling on a SL. The SL was grown by molecular beam epitaxy on a (100)-oriented $n$-doped GaAs substrate. It comprises 15 unit cells, which are separated from two heavily $n$-doped GaAs contacts by Si-doped GaAs layers of width $50 \mathrm{~nm}$ and doping density $1 \times 10^{23} \mathrm{~m}^{-3}$. Each unit cell, of width $d$ and Si doped at $3 \times 10^{22} \mathrm{~m}^{-3}$, is formed by a thin $(1 \mathrm{~nm})$ AlAs barrier and a wide $(7 \mathrm{~nm})$ GaAs quantum well with a 0.8 monolayer InAs insertion at the center. This generates a broad miniband, which is separated from the second miniband by large energy gap [40]. Therefore, according to the classification criteria given in $[12,13]$, our sample is in the miniband conduction regime. For electrical measurements, the SL was processed into circular mesa structures of diameter $20 \mu \mathrm{m}$ with Ohmic contacts to the substrate and top cap layer.
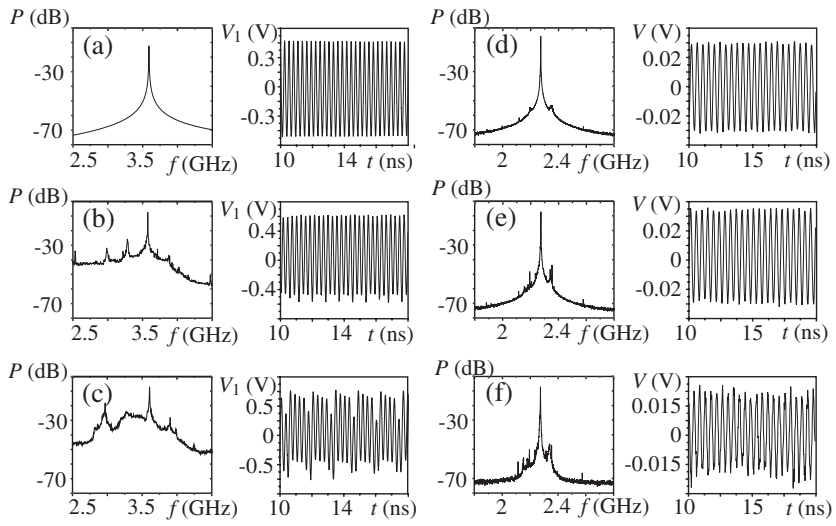

FIG. 5. The numerically (a)-(c) and experimentally (d)-(f) obtained power spectra (left panels) and $V_{1}(t)$ oscillations (right panels) for the SL coupled to two resonators with $V_{0}=(a) 0.42$, (b) 0.43 , and (c) $0.44 \mathrm{~V}$; (d) 0.34, (e) 0.35, and (f) $0.355 \mathrm{~V}$.
The SL was connected to an external high-frequency strip line resonator with a resonant frequency $f_{Q 2}=2.38 \mathrm{GHz}$ (see [37], Figs. S4, S5). Our electrodynamic simulations together with direct measurements showed that the contact bonding to the SL acts like the parasitic resonator, with a resonant frequency of $f_{Q 1}=0.87 \mathrm{GHz}$. A LeCroy SDA 18000 serial data analyzer was used to measure the voltage oscillations generated by the SL. The measurements show periodic $V_{1}(t)$ oscillations with a frequency close to the resonant frequency of the parasitic resonator [22]. However, when the external resonator is connected, the results reveal a transition to chaos. The measurements agree well with our calculations; see Figs. 5(d)-(f). As $V_{0}$ increases, the $V_{1}(t)$ oscillations and their spectra evolve and chaos emerges through the breakdown of quasiperiodic motion. The transition from regular [Fig. 5(d)], through quasiperiodic [Fig. 5(e)], to chaotic oscillations [Fig. 5(f)] demonstrates experimentally that a linear resonator can drive a SL into a chaotic regime [41].

In conclusion, we have shown both theoretically and in experiment that a linear resonator can transform the character of the current-time oscillations and, hence, the associated EM radiation, of a microwave generator. In particular, the presence of a linear resonator counterintuitively induces highly nonlinear phenomena including high-frequency chaotic current oscillations. The chaos appears because the external resonator imposes another oscillatory time scale in the active system, thus inducing complex dynamics via the breakdown of the quasiperiodic motion. We believe that the phenomena of linear resonators described here are applicable to other NDC solid-state devices, including Gunn diodes [42]. The chaotic high-frequency oscillations observed in our experiments may have technological applications in, for example, nonlinear antennas, broadband radar [43-48], and chaos-based logic $[49,50]$. The latter also plays a crucial role in modern chaos-based communication systems [30,31].

This work was partially supported by the President Program (MD-345.2013.2), RFBR (12-02-33071), EPSRC (EP/F005482/1) MES (SSTU-146), and the "Dynasty" Foundation.

*Corresponding author.

F.Kusmartsev@lboro.ac.uk

[1] F. Brennecke, T. Donner, S. Ritter, T. Bourdel, M. Köhl, and T. Esslinger, Nature (London) 450, 268 (2007).

[2] A.M. Zagoskin, Quantum Engineering (Cambridge University Press, Cambridge, England, 2011).

[3] H.-T. Chen, W. J. Padilla, J. M. O. Zide, A. C. Gossard, A. J. Taylor, and R. D. Averitt, Nature (London) 444, 597 (2006).

[4] C. Walther, G. Scalari, M. I. Amanti, M. Beck, and J. Faist, Science 327, 1495 (2010).

[5] M. Khajavikhan, A. Simic, M. Katz, J. H. Lee, B. Slutsky, A. Mizrahi, V. Lomakin, and Y. Fainman, Nature (London) 482, 204 (2012).

[6] M. Pöllinger, D. O'Shea, F. Warken, and A. Rauschenbeutel, Phys. Rev. Lett. 103, 053901 (2009). 
[7] X. R. Huang, D. P. Siddons, A. T. Macrander, R. W. Peng, and X. S. Wu, Phys. Rev. Lett. 108, 224801 (2012).

[8] H. Kroemer, Proc. IEEE 52, 1736 (1964).

[9] J. B. Gunn, IBM J. Res. Dev. 8, 141 (1964).

[10] H. W. Thim, IEEE Trans. Electron Devices 14, 517 (1967); I. V. Altukhov, M. S. Kagan, S. G. Kalashnikov, V. V. Kukushkin, and S. M. Ovechkin, Sov. Tech. Phys. Lett. 6, 237 (1980).

[11] L. Esaki and R. Tsu, IBM J. Res. Dev. 14, 61 (1970).

[12] Andreas Wacker and Antti-Pekka Jauho, Phys. Rev. Lett. 80, 369 (1998).

[13] A. Wacker, Phys. Rep. 357, 1 (2002).

[14] F. Klappenberger, K. N. Alekseev, K. F. Renk, R. Scheuerer, E. Schomburg, S. J. Allen, G. R. Ramian, S. S. Scott, A. Kovsh, V. Ustinov, and A. Zhukov, Eur. Phys. J. B 39, 483 (2004); A. A. Ignatov and V. I. Shashkin, Sov. Phys. JETP66, 526(1987).

[15] Note that active EM properties of the single miniband SLs are distinctly different from QCLs, whose generation involves a population inversion [16-18]. Usually QCLs are engineered to avoid NDC [16]. They are routinely able to generate EM emission within the frequency range from midinfrared to around one $\mathrm{THz}$ [19]. However, for lasing in the sub-THz range QSLs require a low temperature [19] and a very strong magnetic field [20].

[16] J. Faist, F. Capasso, D. L. Sivco, C. Sirtori, A. L. Hutchinson, and A. Y. Cho, Science 264, 553 (1994).

[17] R. Köhler, A. Tredicucci, F. Beltram, H. E. Beere, E. H. Linfield, A. Giles Davies, D. A. Ritchie, R. C. Iotti, and F. Rossi, Nature (London) 417, 156 (2002).

[18] T. Schmielau and M. F. Pereira, Jr, Appl. Phys. Lett. 95, 231111 (2009).

[19] G. Scalari, C. Walther, M. Fischer, R. Terazzi, H. Beere, D. Ritchie, and J. Faist, Laser Photonics Rev. 3, 45 (2009).

[20] A. Wade, G. Fedorov, D. Smirnov, S. Kumar, B. S. Williams, Q. Hu, and J. L. Reno, Nat. Photonics 3, 41 (2009).

[21] T. M. Fromhold et al., Nature (London) 428, 726 (2004).

[22] N. Alexeeva, M. T. Greenaway, A. G. Balanov, O. Makarovsky, A. Patanè, M. B. Gaifullin, F. Kusmartsev, and T. M. Fromhold, Phys. Rev. Lett. 109, 024102 (2012).

[23] M. T. Greenaway, A. G. Balanov, E. Scholl, and T. M. Fromhold, Phys. Rev. B 80, 205318 (2009).

[24] K. Hofbeck et al., Phys. Lett. A 218, 349 (1996); H. Eisele, S. P. Khanna, and E. H. Linfield, Appl. Phys. Lett. 96, 072101 (2010).

[25] C. P. Endres, F. Lewen, T. F. Giesen, S. Schlemmer, D. G. Paveliev, Y. I. Koschurinov, V. M. Ustinov, and A. E. Zhucov, Rev. Sci. Instrum. 78, 043106 (2007); P. Khosropanah, A. Baryshev, W. Zhang, W. Jellema, J. N. Hovenier, J. R. Gao, T. M. Klapwijk, D. G. Paveliev, B. S. Williams, S. Kumar, Q. Hu, J. L. Reno, B. Klein, and J. L. Hesler, Opt. Lett. 34, 2958 (2009); D. G. Paveliev, Y. I. Koshurinov, A. S. Ivanov, A. N. Panin, V. L. Vax, V. I. Gavrilenko, A. V. Antonov, V. M. Ustinov, and A. E. Zhukov, Semiconductors 46, 121 (2012).

[26] K. F. Renk, B. Stahl, A. Rogl, T. Janzen, D. Pavel'ev, Yu. Koshurinov, V. Ustinov, and A. Zhukov, Phys. Rev. Lett. 95, 126801 (2005); A. A. Ignatov, Semicond. Sci. Technol. 26, 055015 (2011).

[27] K. A. Lukin, H. A. Cerdeira, and A. A. Colavita, Appl. Phys. Lett. 71, 2484 (1997); K. A. Lukin, H. A. Cerdeira, and P. P. Maximov, ibid. 83, 4643 (2003).

[28] E. Schöll, Nonlinear Spatio-Temporal Dynamics and Chaos in Semiconductors (Cambridge University Press, Cambridge, England, 2001).
[29] W. Li, I. Reidler, Y. Aviad, Y. Huang, H. Song, Y. Zhang, M. Rosenbluh, and I. Kanter, Phys. Rev. Lett. 111, 044102 (2013).

[30] Special Issue on Applications of Nonlinear Dynamics to Electronic and Information Engineering, edited by $\mathrm{M}$. Hasler et al. [Proc. IEEE. 90, 5 (2002)].

[31] F. C. M. Lau and C. K. Tse, Chaos-Based Digital Communication Systems: Operating Principles, Analysis Methods, and Performance Evaluation (Springer, New York, 2003).

[32] H.-P. Ren, M. S. Baptista, and C. Grebogi, Phys. Rev. Lett. 110, 184101 (2013).

[33] To calculate the charge dynamics in the SL, and thus obtain the current-voltage $I\left(V_{\mathrm{SL}}\right)$ characteristics, we numerically solve the discrete current continuity and Poisson equations.

[34] Yu. A. Romanov, Opt. Spectrosc. 33, 917 (1972).

[35] B. J. Keay, S. Zeuner, S. Allen, K. Maranowski, A. Gossard, U. Bhattacharya, and M. Rodwell, Phys. Rev. Lett. 75, 4102 (1995).

[36] A. A. Koronovskii, A. E. Hramov, V. A. Maximenko, O. I. Moskalenko, K. N. Alekseev, M. T. Greenaway, T. M. Fromhold, and A. G. Balanov, Phys. Rev. B 88, 165304 (2013).

[37] See Supplemental Material at http://link.aps.org/ supplemental/10.1103/PhysRevLett.112.116603 for the Lyapunov analysis, the equivalent circuit of a SL coupled to two resonators, our experimental setup of the SL with external microstrip resonator, the results of our electrodynamic calculations of the mode parameters of the microstrip resonator, and illustrations of the transition from periodic to chaotic dynamics in the SL system.

[38] F. Takens, Lectures Notes in Mathematics (Springer-Verlag, Berlin, 1981), p. 366.

[39] V. S. Anishchenko, Dynamical Chaos-Models and Experiments. Appearance Routes and Structure of Chaos in Simple Dynamical Systems (World Scientific, Singapore, 1995).

[40] A. Patanè, D. Sherwood, L. Eaves, T. M. Fromhold, M. Henini, P. C. Main, and G. Hill, Appl. Phys. Lett. 81, 661 (2002).

[41] The resonator imposes a new oscillatory time scale in the system, thus inducing quasiperiodic current oscillations. Under certain conditions, when the nonlinear mixing [e.g., by nonlinearity in $v_{d}(F)$ dependence] of oscillations with different time scales is strong enough, the quasiperiodic motion loses its stability [39], which leads to the appearance of chaos in the system (see also [37], Fig. S6).

[42] I. V. Altukhov, M. S. Kagan, S. G. Kalashnikov, and V. V. Kukushkin, Sov. Tech. Phys. Lett. 2, 186 (1976); Sov. Phys. Semicond. 13, 1148 (1979).

[43] A. A. Koronovskii, O. I Moskalenko, and A. E Hramov, Phys. Usp. 52, 1213 (2009).

[44] G. S. Nusinovich, A. N. Vlasov, and T. M. Antonsen, Phys. Rev. Lett. 87, 218301 (2001).

[45] V. Dronov, M. R. Hendrey, T. M. Antonsen, and E. Ott, Chaos 14, 30 (2004).

[46] Yu. A. Kalinin, A. A. Koronovskii, A. E. Khramov, E. N. Egorov, and R. A. Filatov, Plasma Phys. Rep. 31, 938 (2005).

[47] B. S. Dmitriev, A. Hramov, A. Koronovskii, A. Starodubov, D. Trubetskov, and Y. Zharkov, Phys. Rev. Lett. 102, 074101 (2009).

[48] R. A. Filatov, A. E. Hramov, Y. P. Bliokh, A. A. Koronovskii, and J. Felsteiner, Phys. Plasmas 16, 033106 (2009).

[49] S. Sinha, and W. L. Ditto, Phys. Rev. Lett. 81, 2156 (1998).

[50] B. Kia, A. Dari, W. L. Ditto, and M. L. Spano, Chaos 21, 047520 (2011). 